\title{
1-JS2-2 Joint Symposium
}

\section{Pharmacological approach to overcome schizophrenia}

\section{Taku Nagai}

Dept. Neuropsychopharmacol. Hosp. Pharm., Nagoya Univ. Grad. Sch. Med.

Schizophrenia is a severe mental illness that affects about $1 \%$ of the population. However, the exact pathoetiology remains unclear and no effective treatment has been available yet. Rare or de novo copy-number variations (CNVs) are likely the most significant contributors to the pathogenesis of schizophrenia. We recently found novel schizophrenia-associated CNVs including ARHGAP10 which encodes a member of the RhoGAP superfamily. ARHGAP10 mutant mice showed an increase in anxiety level. ARHGAP10 mutant mice also manifested potentiation of hyperlocomotion and discriminative cognitive impairment induced by methamphetamine treatment. Morphological analysis revealed that ARHGAP10 mutant mice showed a decrease in neuronal spine density in the medial prefrontal cortex compared to the wild-type mice. Methamphetamine-induced cognitive impairment in ARHGAP10 mutant mice was ameliorated by the treatment with fasudil in dose-dependent manner. These results suggest that mutations in ARHGAP10 increase the risk of schizophrenia, and ARHGAP10 mutant mouse is a novel animal model of schizophrenia based on copy-number variations. Rho signaling pathway may be a potential therapeutic target to develop novel antipsychotics. 